\title{
Entanglement generation through particle detection in systems of identical fermions
}

\author{
P. A. Bouvrie ${ }^{1}$, A. Valdés-Hernández ${ }^{2}$, A. P. Majtey ${ }^{3,4}$, C. Zander ${ }^{5}$, and A. R. Plastino ${ }^{4,6}$ \\ ${ }^{1}$ Centro Brasileiro de Pesquisas Físicas, Rua Dr. Xavier Sigaud 150, Rio de Janeiro, RJ 22290-180, Brazil \\ ${ }^{2}$ Instituto de Física, Universidad Nacional Autónoma de México, \\ Apartado Postal 20-364, México, Distrito Federal, Mexico \\ ${ }^{3}$ Facultad de Matemática, Astronomía y Física, Universidad Nacional de Córdoba, \\ Av. Medina Allende s/n, Ciudad Universitaria, X5000HUA Córdoba, Argentina \\ ${ }^{4}$ Consejo de Investigaciones Científicas y Técnicas de la República Argentina, \\ Av. Rivadavia 1917, C1033AAJ, Ciudad Autónoma de Buenos Aires, Argentina \\ ${ }^{5}$ Physics Department, University of Pretoria, Pretoria 0002, South Africa and \\ ${ }^{6}$ CeBio y Secretaría de Investigaciones, Universidad Nacional del Noroeste de la Prov. de Buenos Aires, \\ UNNOBA-Conicet, Roque Saenz-Peña 456, Junin, Argentina *
}

\begin{abstract}
We investigate the generation of entanglement in systems of identical fermions through a process involving particle detection, focusing on the implications that this kind of processes have for the concept of entanglement between fermionic particles. As a paradigmatic example we discuss in detail a scheme based on a splitting-plus-detection operation. The aim of this scheme is to generate states with a definite number of particles at two separated locations, that can effectively be described as entangled states of two distinguishable qubits, starting from an initial pure state of two indistinguishable fermions exhibiting correlations due purely to antisymmetrization. It is argued that the proposed extraction of a useful entanglement-based resource, given by two distinguishable qubits entangled in the standard sense, does not contravene the notion of entanglement in identicalfermion systems as describing correlations beyond those purely due to their indistinguishability. In point of fact, it is shown that this concept of entanglement, here referred to as fermonic entanglement, actually helps to clarify some essential aspects of the entanglement generation process. In particular, we prove that the amount of entanglement exhibited by the above mentioned pair of distinguishable qubits, obtained after post selection of a state having a definite number of particles in two separated locations, equals the amount of fermionic entanglement created by this detection process. The aforementioned scheme is generalized for the case of $N$-identical fermion systems of arbitrary dimension. It transpires from our present discussion that a proper analysis of entanglement generation during the splitting-plus-detection operation is not only consistent with the concept of fermonic entanglement, but actually reinforces this concept.
\end{abstract}

Keywords: Quantum Entanglement, Fermions, Identical Particles

\section{INTRODUCTION}

The most distinctive feature of quantum systems composed of $N$ identical fermions is that their elementary (antisymmetric) pure state is a single Slater determinant, hence it formally looks entangled (in contrast, for example, with a separable pure state of distinguishable qubits). The ensuing correlations, which we will refer to as 'Slater correlations', can manifest themselves in one or more degrees of freedom. In the present contribution we investigate some aspects of entanglement generation schemes in fermion systems, emphasizing the light they shed on the nature of entanglement between identical fermions and, in particular, on the status of the above mentioned minimal fermion correlations. A concept of entanglement in fermion systems that has attracted considerable attention, and has been applied to the study of diverse quantum systems, is based on the idea that entanglement between identical fermions is associated with the quantum correlations exhibited on top of the Slater correlations [1-17]. This conception of entanglement in fermion systems is relevant, for instance, in atomic physics [18, 19], in quantum chemistry [20], and in the study of quantum dots $[21,22]$. It has also been recently applied to the analysis of the dynamics of interacting fermions performing quantum walks [23]. From this perspective, a single Slater determinant should be considered as a non-entangled state, thus leading to the notion of fermionic entanglement (f-entanglement, for short) to account for the extra correlations beyond those due to the indistinguishability of the parties, and to the antisymmetric property of fermionic states. In the present work we are going to focus mainly on fermionic entanglement. However, it is worth stressing that this is not the only useful concept of entanglement for the study of fermion systems [17, 24-28]. 
Various proposals have been made where quantum states that can be effectively described as entangled states between two distinguishable subsystems at spatially separated locations, are generated starting from minimally (Slater) correlated fermion systems. Here we analyze a scheme that generates entanglement in the above sense, starting from Slater correlations, by means of a splitting-plus-detection operation. Specifically, we consider a system of two electrons located in a double-well potential. An initial single Slater determinant is subjected to a tunneling (or splitting) operation followed by a projective measurement. The spin degrees of freedom of the projected indistinguishable-fermion state are then effectively described by an entangled state of two distinguishable-qubits. We show that such entanglement can be generated only because f-entanglement is also created in the detection process, where a state with a definite number of $\hat{\mathrm{A}}^{\prime}$ particles in each spatially separated location is post-selected. The measurement process and the interaction of the fermionic system with the measuring apparatus is analyzed with the aid of a simple though clarifying model, and a generalization of the splitting process to include systems of $N$ fermions is developed.

Even though most of our present considerations are based on a particular process, the concomitant analysis contributes to elucidate general features of f-entanglement. In particular, it highlights the fact that the amount of entanglement contained in a pure two-fermions state effectively describable as a state of two distinguishable quantum subsystems (that is, with one particle localized at each spatially separated location) is equal to the amount of f-entanglement exhibited by the fermion state. In contrast with previous discussions on entanglement generation with identical particles [27], our analysis clearly identifies the detection process as the origin of the generated f-entanglement, and goes in line with the statement that the Slater correlations do not provide on their own a resource for implementing quantum information processing tasks $[1,2,5,8]$. This is consistent with the possibility of assigning complete sets of properties to the subsystems of the composite system $[1,5]$.

This work is structured as follows. In Sec. II we present a brief review of the definition of f-entanglement in $N$ identical fermion systems. In Sec. III we propose and analyze a scheme of entanglement extraction for systems composed by two identical fermions. We do this by considering a systems of two electrons located in a double-well potential and performing a splitting-plus-detection process acting on an initial non-entangled (in the fermionic sense) state. In Sec. IV we introduced an idealized toy model in order to analize the role of the detection operation. The process for generating entanglement is generalized to systems of $N$ identical fermions in a double-well potential in Sec. V. Finally, some conclusions are drawn in Sec. VI.

\section{FERMIONIC ENTANGLEMENT}

The concept of entanglement for pure states of bipartite quantum systems constituted by to distinguishable subsystems $a$ and $b$ is well known and offers no controversy. Let $|\phi\rangle \in \mathcal{H}_{a} \otimes \mathcal{H}_{b}$ such such a state, where $\mathcal{H}_{a}$, and $\mathcal{H}_{b}$ are the Hilbert spaces associated with the subsystems, and $\mathcal{H}_{a} \otimes \mathcal{H}_{b}$ is the Hilbert space of the composite system. The state is non entangled (separable) if it can be written as the product of states $\left|\psi_{a}\right\rangle \in \mathcal{H}_{a}$ and $\left|\psi_{b}\right\rangle \mathcal{H}_{b}$,

$$
|\phi\rangle=\left|\psi_{a}\right\rangle \otimes\left|\psi_{b}\right\rangle
$$

A state that can not be written in the above way is entangled. The amount of entanglement $\mathcal{E}$ of the state $|\phi\rangle$ can be measured by the von Neuman entropy $S$ of either of the marginal density matrices $\rho_{A, B}=\operatorname{Tr}_{B, A}[|\phi\rangle\langle\phi\rangle \mid]$ associated with the subsystems,

$$
\mathcal{E}[|\phi\rangle\langle\phi\rangle \mid]=S\left[\rho_{A, B}\right]
$$

The above concept of entanglement for pure states of distinguishable subsystems can be extended to composite systems constituted by more that two subsystems. If we have a pure state $\phi \in \mathcal{H}_{a_{1}} \otimes \cdots \otimes \mathcal{H}_{a_{N}}$ of a composite system with $N$ distinguishable subsystems, we say that $\phi$ is fully separable 8 and consequently, has no entanglement at all), if it can be written as

$$
|\phi\rangle=\left|\psi_{a_{1}}\right\rangle \otimes \cdots \otimes\left|\psi_{a_{N}}\right\rangle
$$

with $\left|\psi_{a_{i}}\right\rangle \in \mathcal{H}_{a_{i}}$. We shall refer to the above reviewed concept of entanglement corresponding to pure states of systems with distinguishable subsystems, as the standard concept of entanglement. We shall say that states of systems with distinguishable subsystems that do not comply with (3) are entangled in the standard (that is, referred to distinguishable subsystems) sense. 
The concept of entanglement for systems constituted by $N$ identical fermions is more complex than the corresponding concept for systems with distinguishable subsystems. Indeed, various concepts and measures of entanglement for fermions have been advanced, that are useful for analyzing different aspects of entanglement in fermionic systems. Here we are going to deal mainly with the concept of fermionic entanglement, that we shall explain next. Let us consider a system of $N$ identical fermions with a $d$ dimensional single-particle Hilbert space $\mathcal{H}_{f}$, with $d \geq N$. The space $\mathcal{H}_{f}^{\otimes N}$ of completely anti-symmetric fermion states has then dimension $\frac{d !}{N !(d-N) !}$. Let $\left\{\left|\alpha_{1}\right\rangle,\left|\alpha_{2}\right\rangle, \ldots,\left|\alpha_{N}\right\rangle\right\}$ be a set of $N$ orthonormal states in $\mathcal{H}_{f}$. The antisymmetric combination in $\mathcal{H}_{f}^{\otimes N}$

$$
\left|\psi^{s l}\right\rangle=\frac{1}{\sqrt{N !}} \sum_{\substack{\left\{i_{1}, \ldots, i_{N}\right\} \\ \in S_{p}(1, \ldots, N)}} \varepsilon^{i_{1} \ldots i_{N}}\left|\alpha_{i_{1}}\right\rangle\left|\alpha_{i_{2}}\right\rangle \cdots\left|\alpha_{i_{N}}\right\rangle,
$$

defines what is called a Slater determinant. Here $\varepsilon^{i_{1} \ldots i_{N}}$ stands for the $N$-dimensional Levi-Civita tensor, and $S_{p}(1, \ldots, N)$ are the $N$ ! permutations of the set $\{1,2, \ldots, N\}$. An $N$-identical-fermion state is regarded as separable if and only if its density matrix is of the form [1]

$$
\rho^{s e p}=\sum_{k} p_{k}\left|\psi_{k}^{s l}\right\rangle\left\langle\psi_{k}^{s l}\right|
$$

with $\sum_{k} p_{k}=1$. A state that cannot be decomposed as (5) is regarded as entangled in the fermionic sense, that is, endowed with f-entanglement. In the present work we shall mainly focus on the interplay between f-entanglement, on the one hand, and entanglement in the standard sense between subsystems that are (effectively) distinguishable, on the other hand.

Let now $\{|1\rangle,|2\rangle, \ldots,|d\rangle\}$ stand for an orthonormal basis of the single-particle Hilbert space. It is clear that, out of this $d$ single particle states it is possible to construct $\frac{d !}{N !(d-N) !}$, mutually orthogonal, Slater determinants, that constitutes a basis (Slater basis) of $\mathcal{H}_{f}^{\otimes N}$. This basis, consisting of states with no $f$-entanglement, plays a role similar to that played by the the computational basis when dealing with systems of distinguishable qbits (or qdits).

Another concept of entanglement that is relevant for systems of identical fermions is mode entanglement. Mode entanglement is, formally, given by the same relations as those defining entanglement for distinguishable subsystems, when the fermion systems is expressed in the Fock (mode occupation) representation. When one has a system of fermions in a pure state shared by two parties $A$ and $B$, the amount of mode entanglement $E_{M}$ is given by evaluating (2) for the bipartite state expressed in the mode representation. An intriguing aspect of mode entanglement is that one can have an entangled state even for a system consisting of just one particle. Indeed, consider a particle in an equal quantum superposition of being at two different spatial locations $A$ and $B$. It's state, written in the mode-occupation representation using standard, self-explanatory notation, is,

$$
\frac{1}{\sqrt{2}}(|0,1\rangle+|1,0\rangle)
$$

which certainly looks entangled.

Finally, another relevant concept of entanglement for identical fermions (actually, for identical particles in general) is the one proposed in [26]. This is an operational definition pf entanglement for a system of $N$ identical particles in a pure state $\left|\Psi_{A B}\right\rangle$ shared by two parties $A$ and $B$. It leads to a quantitative measure of entanglement given by,

$$
E_{p}\left[\left|\Psi_{A B}\right\rangle\right]=\sum_{n} P_{n} E_{M}\left[\Psi_{A B}^{(n)}\right],
$$

where $\Psi_{A B}^{(n)}$ is the projection of the state $\Psi_{A B}$ into the subspace corresponding to having a definite number $n$ of particles for $A$ (and $N-n$ for $B$ ). $P_{n}$ is the probability of getting the result $n$ when measuring the number of particles in $A$. The measure (7) is more precise than mode entanglement in assessing the available amount of entanglement, conceived as a real resource to implement quantum information tasks such as teleportation. We shall come back to this issue in the next Section.

\section{ENTANGLEMENT EXTRACTION IN TWO-FERMION SYSTEMS}

We start by considering a system of two electrons located in a double-well potential (e.g., a pair of electrons in coupled quantum dots [29]). The qubits are realized by the spin degree of freedom of the electrons, with states $|\downarrow\rangle$ 
and $|\uparrow\rangle$. Let $|A\rangle$ and $|B\rangle$ denote the spatial part of the state, corresponding to spatially localized wave functions in the left $(A)$ and right $(B)$ well, with $\langle A \mid B\rangle=0$. Then, an orthonormal basis of the (four-dimensional) single-particle state space is $\{|A\rangle|\downarrow\rangle,|A\rangle|\uparrow\rangle,|B\rangle|\downarrow\rangle,|B\rangle|\uparrow\rangle\}$. Initially the two electrons are in the left well, hence

$$
\left|\psi_{\text {init }}\right\rangle=\frac{1}{\sqrt{2}}(|A\rangle|\downarrow\rangle \otimes|A\rangle|\uparrow\rangle-|A\rangle|\uparrow\rangle \otimes|A\rangle|\downarrow\rangle) .
$$

Next, the potential barrier is reduced during a time interval $\tau$, leading to a non-vanishing tunneling amplitude $\sqrt{p}$, with $p=p(\tau) \in[0,1][31,32]$. Then, the potential barrier is raised again. The complete process is thus equivalent to a splitting transformation $U_{\text {split }}=U_{f} \otimes U_{f}$, where the unitary operator $U_{f}$ acting on $\mathcal{H}_{f}$ is such that

$$
U_{f}|A\rangle|\sigma\rangle=\sqrt{1-p}|A\rangle|\sigma\rangle+\sqrt{p}|B\rangle|\sigma\rangle,
$$

where $\sigma$ is either $\uparrow$ or $\downarrow$. The final state reads

$$
\begin{aligned}
\left|\psi_{\text {final }}\right\rangle & =\frac{1}{\sqrt{2}}[(1-p)(|A\rangle|\downarrow\rangle \otimes|A\rangle|\uparrow\rangle-|A\rangle|\uparrow\rangle \otimes|A\rangle|\downarrow\rangle)+\sqrt{p(1-p)}(|A\rangle|\downarrow\rangle \otimes|B\rangle|\uparrow\rangle-|B\rangle|\uparrow\rangle \otimes|A\rangle|\downarrow\rangle \\
& +|B\rangle|\downarrow\rangle \otimes|A\rangle|\uparrow\rangle-|A\rangle|\uparrow\rangle \otimes|B\rangle|\downarrow\rangle)+p(|B\rangle|\downarrow\rangle \otimes|B\rangle|\uparrow\rangle-|B\rangle|\uparrow\rangle \otimes|B\rangle|\downarrow\rangle)] .
\end{aligned}
$$

It is straightforward to verify that $\left|\psi_{\text {final }}\right\rangle$ is a single Slater determinant,

$$
\left|\psi_{\text {final }}\right\rangle=\frac{1}{\sqrt{2}}\left(\left|\xi_{\downarrow}\right\rangle \otimes\left|\xi_{\uparrow}\right\rangle-\left|\xi_{\uparrow}\right\rangle \otimes\left|\xi_{\downarrow}\right\rangle\right)
$$

where $\left|\xi_{\sigma}\right\rangle=U_{f}|A\rangle|\sigma\rangle$. This confirms that no f-entanglement between the particles was created by the splitting operation, and is consistent with the fact that the splitting transformation on the two-fermion system is a unitary operation that is local in $\mathcal{H}_{f} \otimes \mathcal{H}_{f}$ (yet clearly is a spatially-nonlocal operation.) [2].

The terms proportional to $(1-p)$ and $p$ in Eq. (10) correspond to a situation in which both fermions are in the same well, and have no f-entanglement. Yet the term proportional to $\sqrt{p(1-p)}$ exhibits a finite amount of f-entanglement. By projecting (10) onto the state with one particle in each well, we obtain (after normalization)

$$
\left|\psi_{\text {proj }}\right\rangle=\frac{1}{2}(|A\rangle|\downarrow\rangle \otimes|B\rangle|\uparrow\rangle-|B\rangle|\uparrow\rangle \otimes|A\rangle|\downarrow\rangle+|B\rangle|\downarrow\rangle \otimes|A\rangle|\uparrow\rangle-|A\rangle|\uparrow\rangle \otimes|B\rangle|\downarrow\rangle) .
$$

This state cannot be written as a single Slater determinant in any basis, hence it is entangled in the fermionic sense. If now we let an agent (e.g. Alice) in $A$, and an agent (e.g. Bob) in $B$ to have access to the particle in their corresponding well, then Alice and Bob each have a single qubit. These qubits are clearly distinguishable, since each one pertains to a distinguishable physical agent. Therefore, once we ascribe the basis $\mathcal{H}_{A}=\{|A\rangle|\downarrow\rangle,|A\rangle|\uparrow\rangle\}$ to Alice, and the basis $\mathcal{H}_{B}=\{|B\rangle|\downarrow\rangle,|B\rangle|\uparrow\rangle\}$ to Bob, the two-indistinguishable-fermion state (12) becomes effectively equivalent to the two-distinguishable-qubit state,

$$
|\psi\rangle=\frac{1}{\sqrt{2}}\left(|\downarrow\rangle_{A} \otimes|\uparrow\rangle_{B}-|\uparrow\rangle_{A} \otimes|\downarrow\rangle_{B}\right)
$$

where the indices denote, respectively, Alice's and Bob's qubits. Since these two qubits are both localized and individually accessible —unlike the particles in (8) -,$|\psi\rangle$ can be used as a resource for non-trivial quantum information tasks. The state (13) is entangled in the standard sense of entanglement between distinguishable subsystems. Indeed, it is a maximally entangled Bell state [2].

Now, the fact that the state (13) is entangled in the standard sense of entanglement between distinguisable parties, is not unrelated to the fact that the state (12) is entangled in the fermionic sense. In point of fact, as we shall shortly discuss in more detail, the entanglement between the two effectively distinguishable qubits in (13) is inextricably linked to the f-entanglement contained in (12). This last state arose as a result of a detection process. Thus, it follows from these considerations that it is possible to generate entangled states of two effectively distinguishable qubits starting from two identical fermions described by a single Slater determinant, in a way that is fully consistent with the definition of fermionic entanglement explained above. In fact, we can resort to the (fermionic) concurrence introduced in [2], to quantify the f-entanglement between the fermions in Eq. (12). The result is that $\left|\psi_{\text {proj }}\right\rangle$ is a maximally f-entangled state. The maximal f-entanglement of $\left|\psi_{\text {proj }}\right\rangle$ corresponds to the maximal entanglement between the two distinguishable qubits described by $|\psi\rangle$. Indeed, the amount of $f$-entanglement exhibited by $\left|\psi_{\text {proj }}\right\rangle$ is numerically equal to the amount of entanglement of the two-qubits state $|\psi\rangle$. It is important to note that even 
though the state obtained after the splitting (and before detection) has (mode) entanglement with respect to the $A \mid B$ partition, it is not yet a state with a definite number of particles in each of the two spatial locations and, it is not yet suitable for the implementation of standard protocols like teleportation. In order to obtain a state with a definite number of particles in each location, one needs also to perform the detection process which, after post selection of a state with one particle in each location, gives rise to a state endowed with f-entanglement.

The above considerations are related to the operational definition, and concomitant quantitative measure $E_{p}$, advanced by Wiseman and Vaccaro (WV) in [26], for the amount of entanglement between two parties that share a pure state of a system consisting of $N$ indistinguishable particles. An essential ingredient behind the WV proposal is that the mode entanglement between two separated parties is not always fully usable to perform tasks like teleportation. The reason for this limitation is that, if the two parties don't have a definite number of particles, they can not arbitrarily manipulate or measure their local systems without violating the particle number superselection rule (SSR). That is, they are not able to perform the required operations without, at some stage, creating quantum superpositions of states with different numbers of particles, which are forbidden by the particle number SSR. These ideas were later generalized to take into account more general SSR [30]. In the case of the state (10), obtained after the splitting operation, the WV measure (referred to as "accessible entanglement" in the general setting discussed in [30]) is $E_{p}\left[\left|\psi_{\text {final }}\right\rangle\right]=2 p(1-p)$ We see that the value of $E_{p}\left[\left|\psi_{\text {final }}\right\rangle\right]$ is precisely equal to the probability of getting 1 when measuring the number of particles in one of the two locations $A, B$ and, thus, post selecting the state (12) having one particle in each location.

In the general case of two identical fermions with a $(2 n)$-dimensional single-particle Hilbert space, a particledetection process resulting in one particle located at $A$ and the other one located at $B$, leads (irrespective of which was original state before the detection process) to a state of the form

$$
|\psi\rangle=\sum_{i, j=1}^{n} \frac{c_{i, j}}{\sqrt{2}}(|A\rangle|i\rangle \otimes|B\rangle|j\rangle-|B\rangle|j\rangle \otimes|A\rangle|i\rangle),
$$

where the labels $i, j$ correspond to the states of the internal degrees of freedom of the fermions, $\{|i\rangle, i=1, \ldots, n\}$ denotes an orthonormal basis for the associated $n$-dimensional Hilbert space (for instance, for $s$-spin fermions we have $n=2 s+1)$, and $c_{i, j}$ are complex coefficients with $\sum_{i, j}\left|c_{i, j}\right|^{2}=1$. When agents located at $A$ or $B$ operate only upon the internal degree of freedom, the two-fermion state is effectively described by the state

$$
|\psi\rangle_{\mathrm{eff}}=\sum_{i, j=1}^{n} c_{i, j}|i\rangle_{A} \otimes|j\rangle_{B}
$$

of a bipartite system consisting of two distinguishable subsystems, each one with an $n$-dimensional Hilbert space.

If we now consider the Schmidt decomposition of (15),

$$
|\psi\rangle_{\mathrm{eff}}=\sum_{i=1}^{n} \sqrt{\lambda_{i}}\left|\alpha_{i}\right\rangle_{A} \otimes\left|\beta_{i}\right\rangle_{B}
$$

it is verified after some algebra that the fermionic Schmidt-like decomposition of the state (14) is given by

$$
|\psi\rangle=\sum_{i=1}^{n} \sqrt{\frac{\lambda_{i}}{2}}\left(|A\rangle\left|\alpha_{i}\right\rangle \otimes|B\rangle\left|\beta_{i}\right\rangle-|B\rangle\left|\beta_{i}\right\rangle \otimes|A\rangle\left|\alpha_{i}\right\rangle\right) .
$$

It follows from these Schmidt decompositions that the quantitative amount of entanglement (in the sense of entanglement between distinguishable parties) of the effective state (15) coincides with the fermionic amount of entanglement exhibited by the full two-fermion state (14). Using the linear entropy $S=1-\operatorname{Tr} \rho_{f}^{2}$ of the single-particle reduced density matrix $\rho_{f}$ to quantify the amount of entanglement, we get $S=1-\sum_{i} \lambda_{i}^{2}$ both for the full fermionic state (14) and for the effective state (15), a quantity that vanishes if the state (14) is given by a single Slater determinant. Therefore, when a particle-detection process results in a pure state of two fermions localized at different places (with spatially non-overlapping wavefunctions), it is impossible to entanglement between the effectively distinguishable parties, without at the same time generating f-entanglement. Indeed, the amount of entanglement obtained between the distinguishable subsystems is precisely the same as the amount of fermionic entanglement generated.

Comparison of (8) and (13) suggests that the net effect of the splitting-plus-detection process is that of transforming the Slater correlations between the individually unaccessible fermions into entanglement correlations between two 
independently accessible, distinguisable, qubits. We must stress, however, that this interpretation has to be taken with a grain of salt, because the original state (8) does not have f-entanglement, whereas the two-fermion state (12) resulting from the detection operation is f-entangled. It is plain that the finite amount of f-entanglement exhibited by (12) was created during the detection process: it was not originally contained in the state (8).

It is worthwhile to mention that although for operational purposes one is ultimately interested in the state (13), it is possible to experimentally certify that the full two-fermion state created after the detection process is actually (12). In order to do so, once the projective measurement is performed on the state (10) - thus obtaining a state of the form (12) - , we repeat the splitting transformation using Eq. (9) and $U_{f}|B\rangle|\sigma\rangle=\sqrt{1-p}|B\rangle|\sigma\rangle-\sqrt{p}|A\rangle|\sigma\rangle[31,32]$. Then, the probability of finding both fermions in the same mode is found to be $\mathcal{P}_{A A}=\mathcal{P}_{B B}=2 p(1-p)$, whereas the probability of finding a particle in each mode is $\mathcal{P}_{A B}=1-4 p(1-p)$. If, in contrast, the splitting transformation were performed on a state of the form (13) the resulting probabilities would be $\mathcal{P}_{A A}=\mathcal{P}_{B B}=p(1-p)$, and $\mathcal{P}_{A B}=1-2 p(1-p)$. Therefore, by experimental determination of the counting statistics of particles in both modes it can be certified that the state created is $(12)$.

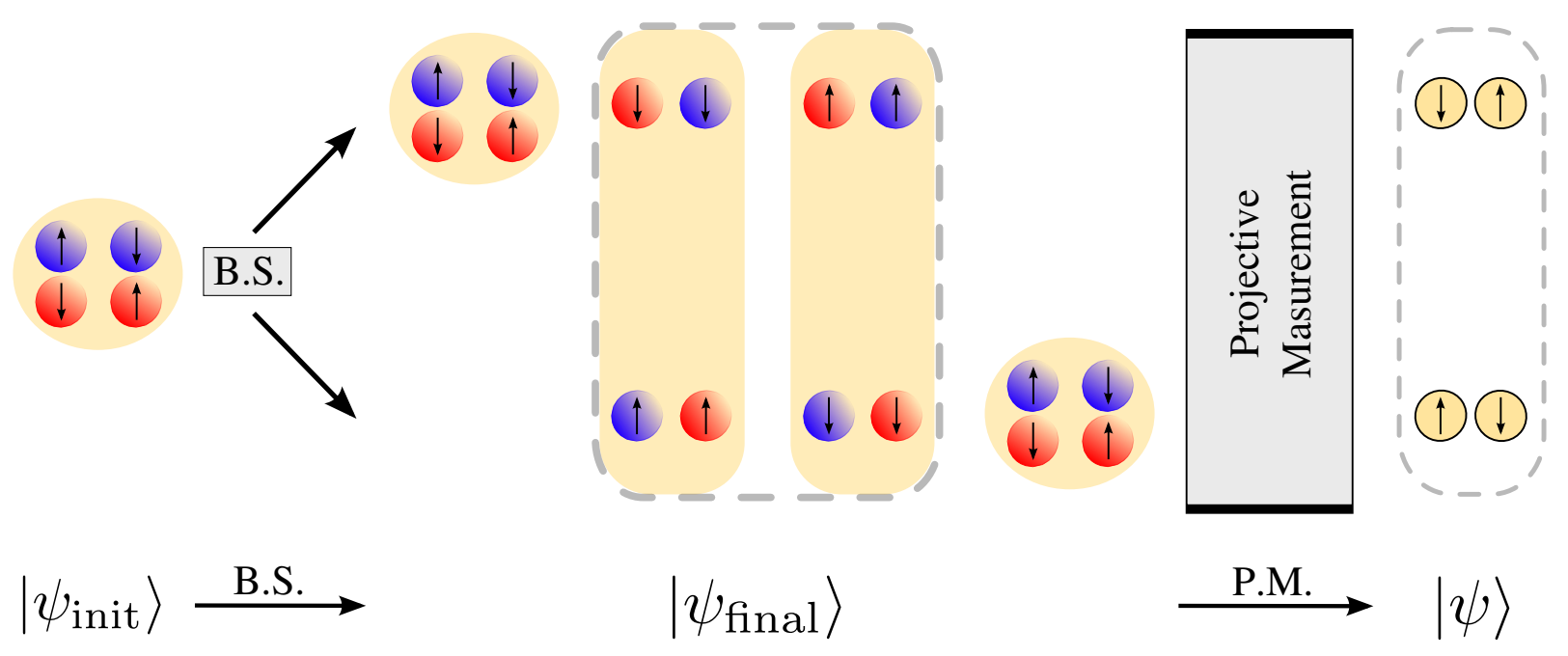

FIG. 1: Outline of the splitting-plus-detection process.

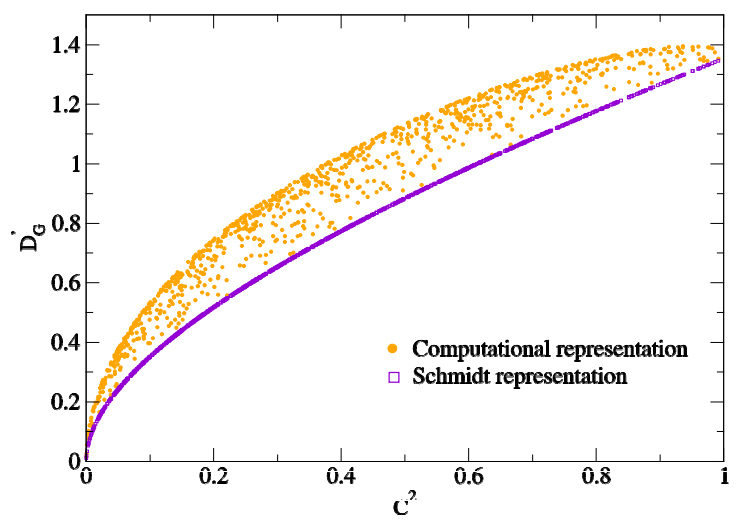

FIG. 2: Entanglement evolution for the state $|2,0\rangle$ under Amplitud Damping decoherence channel. 


\section{INTERACTION OF THE FERMIONIC SYSTEM WITH THE MEASURING APPARATUS}

A fundamental feature of systems of identical parties is that the allowed transformations over the system preserve the exchange-symmetry of the state. An immediate consequence is that when a measurement is performed on a two-fermion system, the interaction between the fermions and the measurement apparatus (or detector) affects each particle in the same way. Thus the Hamiltonian $H_{\text {int }}$ describing the interaction of the two fermions with the apparatus $\mathcal{M}$ has the form [33]

$$
H_{\mathrm{int}}=H_{f M} \otimes \mathbb{I}_{f}+\mathbb{I}_{f} \otimes H_{f M} .
$$

Here $\mathbb{I}_{f}$ denotes the identity operator acting on the single-particle Hilbert space $\mathcal{H}_{f}$, and $H_{f M}$ describes the interaction between one fermion and the detector, and acts on the Hilbert space $\mathcal{H}_{f} \otimes \mathcal{H}_{M}$, where $\mathcal{H}_{M}$ is the Hilbert space associated with the detector.

In the problem at hand the aim of the measurement is to determine how many fermions are, let us say, in the well $A$. We can consider an idealized toy model that captures the essence of the situation. We start by analyzing the interaction between a single fermion and the measuring apparatus. Let us assume that $\mathcal{H}_{M}$ has an orthonormal basis $\{|n\rangle\}$, with $n=0, \pm 1, \pm 2, \ldots$. When the fermion is in the well $A$, and its interaction with the detector is turned on during a time interval $\tau$, the resulting evolution, governed by the unitary operator $U_{\text {int }}=\exp \left(-i H_{\text {int }} \tau / \hbar\right)$, leaves the state of the particle unchanged and changes the state of the detector according to the unitary transformation $|n\rangle \rightarrow|n+1\rangle$. The fermion-detector interaction is local: if the particle is in the well $B$ it does not interact with the detector. Therefore, the interaction changes the state of the detector according to the location of the particle, leaving the state of the particle unaffected (this situation resembles the CNOT gate). If $\left|\psi_{A}\right\rangle=|\psi\rangle|A\rangle$ is a single-fermion state with the particle localized in the well $A, H_{f M}$ acts according to

$$
H_{f M}\left|\psi_{A}\right\rangle|n\rangle=\left|\psi_{A}\right\rangle\left(\sum_{k} c_{n, k}|k\rangle\right),
$$

where the coefficients $\left\{c_{n, k}\right\}$ form an appropriate Hermitian matrix. For a single-fermion state $\left|\psi_{B}\right\rangle=|\psi\rangle|B\rangle$ corresponding to a fermion localized in the well $B$, we have $H_{f M}\left|\psi_{B}\right\rangle|n\rangle=\epsilon_{0}\left|\psi_{B}\right\rangle|n\rangle$. We choose the zero of energy such that $\epsilon_{0}=0$. Then, we have

$$
H_{f M}=|A\rangle\langle A|\left(\sum_{k, n} c_{n, k}|k\rangle\langle n|\right) .
$$

We now turn to the case in which the detector interacts with a two-fermion system, so that the interaction Hamiltonian is of the form (18). The initial state of the detector is $|0\rangle$; the interaction is then turned on during a time interval $\tau$. If both fermions are localized at the well $B$ (as, for example, in the state $\left|\Phi_{0}\right\rangle=\frac{1}{\sqrt{2}}(|B\rangle|\downarrow\rangle \otimes|B\rangle|\uparrow\rangle-|B\rangle|\uparrow\rangle \otimes|B\rangle|\downarrow\rangle)$, one has $U_{\text {int }}\left|\Phi_{0}\right\rangle|0\rangle=\left|\Phi_{0}\right\rangle|0\rangle$. If there is only one fermion in the well $A$ (as happens, for example, in the state $\left|\Phi_{1}\right\rangle=\frac{1}{\sqrt{2}}(|A\rangle|\downarrow\rangle \otimes|B\rangle|\uparrow\rangle-|B\rangle|\uparrow\rangle \otimes|A\rangle|\downarrow\rangle)$, it can be verified that $H_{\text {int }}$ satisfies $H_{\text {int }}\left|\Phi_{1}\right\rangle|n\rangle=\left|\Phi_{1}\right\rangle\left(\sum_{k} c_{n, k}|k\rangle\right)$. Therefore, the associated time evolution operator $U_{\text {int }}$ yields a transformation similar to the one obtained when having only one particle interacting with the detector, and $U_{\text {int }}\left|\Phi_{1}\right\rangle|0\rangle=\left|\Phi_{1}\right\rangle|1\rangle$. If instead the two fermions are in the well $A$ (as, for example, in the state $\left|\Phi_{2}\right\rangle=\frac{1}{\sqrt{2}}(|A\rangle|\downarrow\rangle \otimes|A\rangle|\uparrow\rangle-|A\rangle|\uparrow\rangle \otimes|A\rangle|\downarrow\rangle)$, we have $H_{\text {int }}\left|\Phi_{2}\right\rangle|n\rangle=\left|\Phi_{2}\right\rangle\left(\sum_{k} 2 c_{n, k}|k\rangle\right)$. In this case, the time evolution operator corresponding to $H_{\text {int }}$ and a time interval $\tau$ has the same effect upon the detector as the evolution operator corresponding to $H_{f M}$ and a time interval $2 \tau$. This effect is therefore the same as that of applying twice the transformation $|n\rangle \rightarrow|n+1\rangle$, yielding $|n\rangle \rightarrow|n+2\rangle$. In summary, the resulting transformation is $U_{\text {int }}\left|\Phi_{2}\right\rangle|0\rangle=\left|\Phi_{2}\right\rangle|2\rangle$. In all cases the number of particles localized in the well $A$ can be read in the final state of the detector.

Consider now a two-fermion state that does not have a definite number of particles in the well $A$. An example of such state is $\left|\psi_{\text {final }}\right\rangle$, given by Eq. (10). According to the above, the interaction between the fermions and the measurement apparatus leads to $U_{\text {int }}\left|\psi_{\text {final }}\right\rangle|0\rangle=|\Psi\rangle$, with

$$
\begin{aligned}
|\Psi\rangle & =\frac{(1-p)}{\sqrt{2}}(|A\rangle|\downarrow\rangle \otimes|A\rangle|\uparrow\rangle-|A\rangle|\uparrow\rangle \otimes|A\rangle|\downarrow\rangle)|2\rangle+\frac{\sqrt{p(1-p)}}{\sqrt{2}}[|A\rangle|\downarrow\rangle \otimes|B\rangle|\uparrow\rangle-|B\rangle|\uparrow\rangle \otimes|A\rangle|\downarrow\rangle \\
& +|B\rangle|\downarrow\rangle \otimes|A\rangle|\uparrow\rangle-|A\rangle|\uparrow\rangle \otimes|B\rangle|\downarrow\rangle]|1\rangle+\frac{p}{\sqrt{2}}(|B\rangle|\downarrow\rangle \otimes|B\rangle|\uparrow\rangle-|B\rangle|\uparrow\rangle \otimes|B\rangle|\downarrow\rangle)|0\rangle .
\end{aligned}
$$

Equation (21) represents the state of the tripartite system after the interaction with the measurement apparatus, and before the result of the measurement is actually read. Clearly, and as a result of the interaction, the detector 
becomes entangled with the fermionic system. According to the discussion below Eq. (12), projection of $|\Psi\rangle$ onto the state $|1\rangle$ of the detector results in an f-entangled two-fermion pure state. If no projection is performed, the reduced two-fermion state $\rho_{f f}=\operatorname{Tr}_{\mathcal{M}}|\Psi\rangle\langle\Psi|$ is a mixed state, whose f-entanglement can be obtained by direct calculation of the (fermionic) concurrence $C\left(\rho_{f f}\right)$, which quantifies the f-entanglement between two fermions whose single-particle Hilbert space has dimension 4 , in a general (pure or mixed) state $\rho_{f f}[2]$. The resulting fermionic concurrence is $C\left(\rho_{f f}\right)=0$, meaning that if no projection is performed (i.e., before the detector clicks), the fermions do not get f-entangled. Therefore, even though the interaction of the fermions with the measuring apparatus is essential for creating the fermionic entanglement, this entanglement cannot be actually extracted until the detector clicks on 1. The moral of these observations is that when dealing with identical parties the measurement of the number of particles present at a given spatial location must not be considered as "neutral" or cost-free regarding fermionic entanglement. The fact that f-entanglement can be created in these processes is hardly more surprising than the fact that entanglement (in the standard sense referred to distinguishable parties) can be created when measuring the Bell operator on a two-qubit system.

\section{ENTANGLEMENT EXTRACTION IN $N$-FERMION SYSTEMS}

The process just discussed has as its final result a state that can be construed as describing two effectively distinguishable subsystems, at separated spatial locations, and each having a definite number of particles. This final state is entangled in the standard (distinguishable parties) sense, and can be directly used to implement quantum-information tasks such as teleportation. This final state, that clearly constitutes an entanglement resource ready to be used, was obtained (after some labour) from an initial state of two electrons, described by a single Slater determinant. In this Section we shall consider a generalization of this process to systems composed of $N$ identical fermions in a double-well potential. An initial Slater determinant of the form (4), corresponding to a situation in which all $N$ fermions are in the well $A$, transforms into another Slater determinant (a separable state in the fermionic sense) once the splitting transformation is performed. However, projection of the evolved state onto states of fixed number of particles in each well (e.g., $M$ in mode $A$ and $N-M$ in mode $B$, with $1 \leq M \leq N-1$ ), leads to a state that is entangled in the fermionic sense. This fermionic entanglement created in the whole process (splitting plus detection) can be evaluated using the measure introduced in [34]. When Alice and Bob operate upon the internal degrees of freedom of the fermions, they share a state with an amount of entanglement (in the standard, distinguishable-party sense) that coincides with the Slater correlations of the initial state, a result similar to the one obtained for bosons in [27].

As a first step to investigate this generalization we decompose a Slater determinant into its Schmidt form. The Slater determinant can be written as

$$
\begin{aligned}
\left|\psi^{s l}\right\rangle & =\frac{1}{\sqrt{N !}} \sum_{\substack{\left\{i_{1}, \ldots, i_{N}\right\} \\
\in S_{p}(1, \ldots, N)}} \varepsilon^{i_{1} \ldots i_{N}}\left|i_{1}, i_{2}, \ldots, i_{N}\right\rangle \\
& =\hat{f}_{1}^{\dagger} \cdots \hat{f}_{N}^{\dagger}|0\rangle=\frac{1}{\sqrt{N !}} \mathcal{A}(|1,2, \ldots, N\rangle)
\end{aligned}
$$

where we resorted to the second quantization notation, in which $\hat{f}_{i}^{\dagger}$ is the fermionic creation operator [2], and $\mathcal{A}$ denotes an (unnormalized) antisymmetric vector, defined according to Eq. (22).

In order to write $\left|\psi^{s l}\right\rangle$ in its Schmidt form, we notice that it can be expressed as

$$
\left|\psi^{s l}\right\rangle=\frac{1}{\sqrt{N !}} \sum_{\substack{\left\{i_{1}, \ldots, i_{N}\right\} \\ \in S_{p}^{(M)}(1, \ldots, N)}} \varepsilon^{i_{1} \ldots i_{N}} \mathcal{A}\left(\left|i_{1}, i_{2}, \ldots, i_{M}\right\rangle\right) \mathcal{A}\left(\left|i_{M+1}, \ldots, i_{N}\right\rangle\right)
$$

where $S_{p}^{(M)}(1, \ldots, N)$ denotes the $\left(\begin{array}{l}N \\ M\end{array}\right)$ different ways of choosing the first $M$ indices with $i_{1}<\cdots<i_{M}$ from the set $\{1, \ldots, N\}$. The condition $i_{M+1}<\ldots<i_{N}$ sets the remaining $N-M$ indices of the second antisymmetric product state of Eq. (23). We now write

$$
\frac{1}{\sqrt{n !}} \mathcal{A}\left(\left|i_{1}, i_{2}, \ldots, i_{n}\right\rangle\right)=\left|\psi_{i_{1}, \ldots, i_{n}}^{s l}\right\rangle
$$


so that Eq. (23) decomposes into its Schmidt form

$$
\left|\psi^{s l}\right\rangle=\sum_{\substack{\left\{i_{1}, \ldots, i_{N}\right\} \\ \in S_{p}^{(M)}(1, \ldots, N)}} \alpha_{i_{1} \ldots i_{N}}\left|\psi_{i_{1}, \ldots, i_{M}}^{s l}\right\rangle\left|\psi_{i_{M+1}, \ldots, i_{N}}^{s l}\right\rangle,
$$

with

$$
\alpha_{i_{1} \ldots i_{N}}=\left(\begin{array}{l}
N \\
M
\end{array}\right)^{-\frac{1}{2}} \varepsilon^{i_{1} \ldots i_{N}}
$$

being the Schmidt coefficients, and $\left|\psi_{i_{1}, \ldots, i_{M}}^{s l}\right\rangle\left|\psi_{i_{M+1}, \ldots, i_{N}}^{s l}\right\rangle$ their corresponding eigenvectors. In this way an $N$-fermion Slater determinant decomposes into the sum of products of $M$ - and $(N-M)$ - fermion Slater determinants, that result from a particular bipartition $(M: N-M)$ of the complete system. For such a bipartition, the Schmidt rank of a single $N$-fermion Slater determinant is thus $\mathcal{S}_{s l}^{(M)}=\left(\begin{array}{l}N \\ M\end{array}\right)$. From now on we shall denote by $\mathcal{S}_{\phi}^{(M)}$ the Schmidt rank associated with the $(M: N-M)$ partition effected upon the $N$-fermion system in the state $|\phi\rangle\left(\mathcal{S}_{s l}^{(M)}\right.$ corresponding to the particular case of a global Slater state).

We now consider an initial state separable in the fermionic sense (Slater determinant) in which all $N$ fermions are in the well $A$. Thus what we previously called state $\left|i_{n}\right\rangle$ will be substituted by $\left|A_{i_{n}}\right\rangle=|A\rangle\left|i_{n}\right\rangle$. The splitting operation associated to the unitary transformation in Eq. (4) corresponds to the following map involving the fermionic creation operators,

$$
\hat{f}_{A_{i_{n}}}^{\dagger} \rightarrow \sqrt{(1-p)} \hat{f}_{A_{i_{n}}}^{\dagger}+\sqrt{p} \hat{f}_{B_{i_{n}}}^{\dagger}
$$

Under this operation the initial state

$$
\left|\psi_{\text {init }}\right\rangle=\left|\psi_{A_{1}, \ldots, A_{N}}^{s l}\right\rangle=\hat{f}_{A_{1}}^{\dagger} \hat{f}_{A_{2}}^{\dagger} \ldots \hat{f}_{A_{N}}^{\dagger}|0\rangle
$$

transforms into

$$
\left|\psi_{\text {final }}\right\rangle=\sum_{M=0}^{N} \sqrt{(1-p)^{M} p^{N-M}} \sum_{\substack{\left\{i_{1}, \ldots, i_{N}\right\} \\ \in S_{p}^{(M)}(1, \ldots, N)}} \varepsilon^{i_{1} \ldots i_{N}} \hat{f}_{A_{i_{1}}}^{\dagger} \ldots \hat{f}_{A_{i_{M}}}^{\dagger} \hat{f}_{B_{i_{M}+1}}^{\dagger} \ldots \hat{f}_{B_{i_{N}}}^{\dagger}|0\rangle,
$$

where the sum in the last line runs over the $\left(\begin{array}{l}N \\ M\end{array}\right)$ different ways of distributing the $M$ (out of $N$ ) indices $i_{1}, \ldots, i_{M}($ taken from the set $\{1, \ldots, N\}$ ) among the $A$ 's. The state (29) is a single Slater determinant, hence is non-entangled in the fermionic sense. This follows from the fact that the splitting transformation does not create fermionic entanglement, as discussed below Eq. (4), and can be easily verified by writing the initial state as

$$
\left|\psi_{\text {init }}\right\rangle=\frac{1}{\sqrt{N !}} \sum_{\substack{\left\{i_{1}, \ldots, i_{N}\right\} \\ \in S_{p}(1, \ldots, N)}} \varepsilon^{i_{1} \ldots i_{N}}\left|A_{i_{1}}, \ldots, A_{i_{N}}\right\rangle
$$

so that under the splitting operation,

$$
\left|A_{i_{n}}\right\rangle=|A\rangle\left|i_{n}\right\rangle \rightarrow\left|\chi_{i_{n}}\right\rangle=(\sqrt{(1-p)}|A\rangle+\sqrt{p}|B\rangle)\left|i_{n}\right\rangle
$$

$\left|\psi_{\text {init }}\right\rangle$ transforms into

$$
\left|\psi_{\text {final }}\right\rangle=\frac{1}{\sqrt{N !}} \sum_{\substack{\left\{i_{1}, \ldots, i_{N}\right\} \\ \in S_{p}(1, \ldots, N)}} \varepsilon^{i_{1} \ldots i_{N}}\left|\chi_{i_{1}}, \ldots, \chi_{i_{N}}\right\rangle .
$$

Let us now project (29) onto states of fixed number of particles in each spatial mode $(M$ in mode $A, N-M$ in mode $B$ ), thus obtaining

$$
\begin{aligned}
\left|\psi_{\text {proj }}\right\rangle= & \underset{\substack{\left\{i_{1}, \ldots, i_{N}\right\} \\
\in}}{\mathcal{N} \varepsilon^{i_{1} \ldots i_{N}}} \hat{f}_{A_{i_{1}}}^{\dagger} \ldots \hat{f}_{A_{i_{M}}}^{\dagger} \hat{f}_{B_{i_{M}+1}}^{\dagger} \ldots \hat{f}_{B_{i_{N}}}^{\dagger}|0\rangle \\
= & \mathcal{N} \sum_{\substack{\left\{i_{1}, \ldots, i_{N}\right\} \\
\in i^{(M)}(1, \ldots, N)}} \varepsilon^{i_{1} \ldots i_{N}}\left|\psi_{A_{i_{1}}, \ldots, A_{i_{M}}, B_{i_{M+1}}, \ldots, B_{i_{N}}}^{s l}\right\rangle, \\
& \in S_{p}^{(M)}(1, \ldots, N)
\end{aligned}
$$


with $\mathcal{N}$ a normalization factor. Since each term in the sum involves one of the $\left(\begin{array}{l}N \\ M\end{array}\right)$ different ways of distributing $M$ indices $i_{n}$ (with $\left\{i_{n}\right\}=\{1, \ldots, N\}$ ) among the $A$ 's (which fixes the remaining $N-M$ indices appearing in the $B$ 's $),\left|\psi_{\text {proj }}\right\rangle$ is a linear combination of Slater determinants, each of which differs from any other in at least one pair of creation operators $\hat{f}_{A_{i_{n}}}^{\dagger} \hat{f}_{B_{i_{m}}}^{\dagger}$. Hence, the $\left(\begin{array}{l}N \\ M\end{array}\right)$ Slater determinants appearing in Eq. (32) are all orthogonal, which fixes $\mathcal{N}=\left(\begin{array}{l}N \\ M\end{array}\right)^{-1 / 2}$. Using Eq. (26), $\left|\psi_{\text {proj }}\right\rangle$ rewrites as

$$
\left|\psi_{\text {proj }}\right\rangle=\sum_{\substack{\left\{i_{1}, \ldots, i_{N}\right\} \\ \in S_{p}^{(M)}(1, \ldots, N)}} \alpha_{i_{1} \ldots i_{N}}\left|\psi_{A_{i_{1}}, \ldots, A_{i_{M}}, B_{i_{M+1}}, \ldots, B_{i_{N}}}^{s l}\right\rangle .
$$

In what follows we will demonstrate that, unlike $(29),\left|\psi_{\text {proj }}\right\rangle$ is entangled in the fermionic sense. This can be verified by performing its Schmidt decomposition. To do that, we first resort to Eq. (25) to expand each of the Slater states appearing in the sum (33) into a superposition of two Slater determinants with fixed number of particles, namely $M$ for the first one and $N-M$ for the second, thus obtaining

$$
\left|\psi_{\text {proj }}\right\rangle=\sum_{\substack{\left\{i_{1}, \ldots, i_{N}\right\} \\ \in S_{p}^{(M)}(1, \ldots, N) \in S_{p}^{(M)}\left(A_{i_{1}}, \ldots, A_{i_{M}}, B_{i_{M+1}}, \ldots, B_{i_{N}}\right)}} \sum_{\substack{\left\{j_{1}, \ldots, j_{N}\right\} \\ i_{1} \ldots i_{N}}} \alpha_{j_{1} \ldots j_{N}}\left|\psi_{j_{1}, \ldots, j_{M}}^{s l}\right\rangle\left|\psi_{j_{M+1}, \ldots, j_{N}}^{s l}\right\rangle .
$$

It is worth mentioning that Eq. (34), describing the sum of Slater determinants each one in its Schmidt form, does not represent in general the Schmidt form of the state $\left|\psi_{\text {proj }}\right\rangle$. In order to obtain such a representation we expand the second sum of Eq. (34) into a sum in which each Slater has a fixed number of particles in the modes $A$ and $B$, that is,

$$
\begin{aligned}
& \left|\psi_{\text {proj }}\right\rangle=\sum_{\substack{\left\{i_{1}, \ldots, i_{N}\right\} \\
\in S_{p}^{(M)}(1, \ldots, N)}} \alpha_{i_{1} \ldots i_{N}} \sum_{n=0}^{M} \sum_{\substack{\left\{j_{1}, \ldots, j_{M}\right\} \\
\in S_{p}^{(n)}\left(i_{1}, \ldots, i_{M}\right)}} \sum_{\substack{\left\{j_{M}+1, \ldots, j_{N}\right\} \\
\in S_{p}^{(M-n)}\left(i_{M+1}, \ldots, i_{N}\right)}} \alpha_{j_{1} \ldots j_{N}} \\
& \times\left|\psi_{A_{j_{1}}, \ldots, A_{j_{n}}, B_{j_{M+1}}, \ldots, B_{j_{2 M-n}}}^{s l}\right\rangle\left|\psi_{A_{j_{n+1}}, \ldots, A_{j_{M}}, B_{j_{2 M-n+1}, \ldots, B_{j_{N}}}}^{s l}\right\rangle,
\end{aligned}
$$

where $n$ denotes the number of particles located in mode $A$ in the first Slater. The indices $i$ 's and $j$ 's appearing in the other three sums of Eq. (35) can be reordered as follows:

$$
\begin{aligned}
& \left|\psi_{\text {proj }}\right\rangle=\sum_{n=0}^{M} \sum_{\substack{\left\{i_{1}, \ldots, i_{N}\right\} \\
\in S_{p}^{(n)}(1, \ldots, N) \in S_{p}^{(M-n)}}} \sum_{\substack{\left\{j_{1}, \ldots, j_{N-n}\right\} \\
\left(i_{n+1}, \ldots, i_{N}\right)}} \alpha_{i_{1} \ldots i_{n}, j_{1} \ldots j_{N-n}} \sum_{\substack{\left\{k_{1}, \ldots, k_{N-M}\right\} \\
\in S_{p}^{(M-n)}\left(j_{M}-n+1, \ldots, j_{N-n}\right)}} \alpha_{i_{1} \ldots i_{n}, j_{1} \ldots j_{M-n}, k_{1} \ldots k_{N}-M} \\
& \times\left|\psi_{A_{i_{1}}, \ldots, A_{i_{n}}, B_{j_{1}}, \ldots, B_{j_{M-n}}}^{s l}\right\rangle\left|\psi_{A_{k_{1}}, \ldots, A_{k_{M-n}}, B_{k_{M-n+1}}, \ldots, B_{k_{N-M}}}\right\rangle .
\end{aligned}
$$

Because of the symmetry, we consider $1 \leq M \leq N-M$ hereafter. From this condition it follows that the first Slater state in Eq. (36), with $M$ particles, is already written in the Schmidt basis, since its corresponding Hilbert space has a dimension which is smaller or equal than the one corresponding to the second Slater determinant. Now, for fixed $n$, we introduce a global index $\mathbf{i}_{n}$ to represent the indices $i$ 's and $j$ 's appearing in the first two sums of Eq. (36). This global index denotes the $\mathcal{M}=\frac{N !}{n !(M-n) !(N-M) !}$ ways of partitioning the $N$ distinct single-fermion states $\{1, \ldots, N\}$ into the three the sets: $\left\{i_{1}, \ldots, i_{n}\right\},\left\{j_{1}, \ldots, j_{M-n}\right\}$, and $\left\{j_{M-n+1}, \ldots, j_{N-n}\right\}$, with $n, M-n$ and $N-M$ elements, respectively. In terms of the new index we write the first Slater determinant in Eq. (36) as

$$
\left|\psi_{\mathbf{i}_{n},(n, M-n)}\right\rangle \equiv\left|\psi_{A_{i_{1}}, \ldots, A_{i_{n}}, B_{j_{1}}, \ldots, B_{j_{M-n}}}^{s l}\right\rangle
$$

Since the last sum in (36) runs only over the indices $k$ of the second Slater, the Schmidt basis of the $(N-M)$-fermion subsystem is

$$
\left|\psi_{\mathbf{i}_{n},(M-n, N-2 M+n)}\right\rangle \equiv\left(\begin{array}{c}
N-M \\
M-n
\end{array}\right)_{\substack{\left.\in S_{p}^{(M-n)}\left(k_{1}, \ldots, k_{N-M}\right\} \\
j_{M-n+1}, \ldots, j_{N-n}\right)}}^{-\frac{1}{2}} \varepsilon^{i_{1} \ldots i_{n} j_{1} \ldots j_{M-n} k_{1} \ldots k_{N-M}}\left|\psi_{A_{k_{1}}, \ldots, A_{k_{M-n}}, B_{k_{M-n+1}, \ldots, B_{k_{N}-M}}}^{s l}\right\rangle .
$$


Finally, the Schmidt decomposition of the projected state reads

$$
\left|\psi_{\text {proj }}\right\rangle=\sum_{n=0}^{M} \sum_{\mathbf{i}_{\mathbf{n}}} \lambda_{\mathbf{i}_{n}}\left|\psi_{\mathbf{i}_{n},(n, M-n)}\right\rangle\left|\psi_{\mathbf{i}_{n},(M-n, N-2 M+n)}\right\rangle,
$$

with

$$
\lambda_{\mathbf{i}_{n}}=\varepsilon^{i_{1} \ldots i_{n} j_{1} \ldots j_{N-n}}\left(\begin{array}{c}
N-M \\
M-n
\end{array}\right)^{\frac{1}{2}}\left(\begin{array}{c}
N \\
M
\end{array}\right)^{-1} .
$$

In [34], a pure $N$-fermion state $|\phi\rangle$ was considered, and it was shown that for any bipartition of the form $M:(N-M)$, the $M$-fermion reduced density matrix satisfies $\operatorname{Tr} \rho_{M}^{2} \leq\left(\begin{array}{l}N \\ M\end{array}\right)^{-1}$. It was further demonstrated that the equal sign holds if and only if $|\phi\rangle$ is a Slater determinant. On the other hand, by following the same arguments as exposed in [9], the $M$-fermion reduced density matrix fulfils $\rho_{M}^{2}=\left(\mathcal{S}_{\phi}^{(M)}\right)^{-1} \rho_{M}$ if and only if the state $|\phi\rangle$ is a single Slater determinant. Therefore, from these two conditions, it follows that the state $|\phi\rangle$ is f-entangled if and only if its Schmidt rank $\mathcal{S}_{\phi}^{(M)}$ is larger than $\mathcal{S}_{s l}^{(M)}$. The state (39) is in the Schmidt decomposition form for the bipartition $M: N-M$, and it has Schmidt rank

$$
\mathcal{S}_{\text {proj }}^{(M)}=\sum_{n=0}^{M} \frac{N !}{n !(M-n) !(N-M) !} .
$$

Since,

$$
\mathcal{S}_{\text {proj }}^{(M)}\left(\begin{array}{l}
N \\
M
\end{array}\right)^{-1}=\sum_{n=0}^{M} \frac{M !}{n !(M-n) !}=\sum_{n=0}^{M}\left(\begin{array}{c}
M \\
n
\end{array}\right)=2^{M}>1,
$$

it holds that $\mathcal{S}_{\text {proj }}^{(M)}>\left(\begin{array}{l}N \\ M\end{array}\right)$, and therefore we conclude that the state $\left|\psi_{\text {proj }}\right\rangle$ is indeed entangled in the fermionic sense.

On the other hand, the eigenvalues of the reduced ( $M$-fermion) density matrix

$$
\begin{aligned}
\rho_{M} & =\operatorname{Tr}_{N-M}\left|\psi_{\text {proj }}\right\rangle\left\langle\psi_{\text {proj }}\right| \\
& =\sum_{n=0}^{M} \sum_{\mathbf{i}_{\mathbf{n}}} \lambda_{\mathbf{i}_{n}}^{2}\left|\psi_{\mathbf{i}_{n},(n, M-n)}\right\rangle\left\langle\psi_{\mathbf{i}_{n},(n, M-n)}\right|
\end{aligned}
$$

are given by $\lambda_{\mathbf{i}_{n}}^{2}$, and consequently

$$
\begin{aligned}
\operatorname{Tr} \rho_{M}^{2} & =\sum_{n=0}^{M} \sum_{\mathbf{i}_{\mathbf{n}}} \lambda_{\mathbf{i}_{n}}^{4}=\sum_{n=0}^{M} \sum_{\mathbf{i}_{\mathbf{n}}}\left(\begin{array}{c}
N-M \\
M-n
\end{array}\right)^{2}\left(\begin{array}{l}
N \\
M
\end{array}\right)^{-4} \\
& =\sum_{n=0}^{M} \frac{N !}{n !(M-n) !(N-M) !}\left(\begin{array}{c}
N-M \\
M-n
\end{array}\right)^{2}\left(\begin{array}{c}
N \\
M
\end{array}\right)^{-4}
\end{aligned}
$$

With this expression, the amount of fermionic entanglement generated in the whole process (splitting plus detection) can be determined resorting to the measure introduced in [34].

We now let independent agents in $A$ and $B$ have access to the particles in their corresponding wells, so that Alice and Bob have $M$ and $N-M$ particles, respectively. Notice that though Alice's particles are indistinguishable among themselves, they all are effectively distinguishable from Bob's. With this, the $N$-fermion Hilbert space $\mathcal{H}=\mathcal{H}_{f} \otimes N$ splits into $\mathcal{H}_{A} \otimes \mathcal{H}_{B}$. Therefore, by projecting (33) onto states of the form $\left|A_{j_{1}}, \ldots, A_{j_{M}}\right\rangle \otimes\left|B_{j_{M+1}}, \ldots, B_{j_{N}}\right\rangle$ we get

$$
\begin{aligned}
& |\psi\rangle=\sum_{\substack{\left\{i_{1}, \ldots, i_{N}\right\} \\
\in S_{p}^{(M)}(1, \ldots, N)}} \alpha_{i_{1} \ldots i_{N}}\left|i_{1}, \ldots, i_{M}\right\rangle_{A} \otimes\left|i_{M+1}, \ldots, i_{N}\right\rangle_{B}, \\
& \text {. }
\end{aligned}
$$

where the subindices $A$ and $B$ have the same meaning as those in Eq. (8). The state $|\psi\rangle$, with $\operatorname{Tr} \rho_{A}^{2}=\left(\begin{array}{l}N \\ M\end{array}\right)^{-1}$, thus corresponds to an entangled state shared by two distinguishable entities (Alice and Bob), hence it is legitimately entangled in the usual (distinguishable-party) sense. This entanglement is manifested in the internal degrees of freedom, whose states are represented by the vectors $\left\{\left|i_{n}\right\rangle\right\}$. This effective entanglement between the particles of both modes is the same as the correlations between $M$ and $N-M$ particles due to the antisymmetry of a single-Slater state of $N$ identical fermions, in consonance with results obtained in [27] for bosonic systems. 


\section{CONCLUSIONS}

Our aim in the present work was to examine the surprising fact that useful entanglement between distinguishable and spatially separated parties, having a definite number of particles, can be obtained from a pure state of $N$ identical fermions exhibiting only Slater correlations (i.e., correlations due purely to antisymmetrization), even though deep theoretical reasons have been advanced over the years for considering such a state as non-entangled. To that end we performed a critical analysis of an entanglement generating scheme based on a splitting-plus-detection operation acting on an initial Slater state. Our analysis highlights the role of the detection process. We argue that entanglement between distinguishable, localized parties, each of them having a definite number of particles at their respective locations, can be obtained after the projective measurement only because a state entangled in the fermionic sense also arises as a result of this operation. In fact, we show that the aforementioned entanglement, between distinguishable parties with definite number of particles, cannot be obtained without generating at the same time fermionic entanglement. Moreover, the final quantitative amount of entanglement obtained between the two distinguishable and localized subsystems, is equal to the amount of fermionic entanglement generated. This implies that the fermionic entanglement, which only appears after the measurement process, is essential for a complete understanding of the origin of an entanglement-based resource ready to be used, for instance, to implement tasks like teleportation. It would be interesting to extend the present analysis to the case of initial mixed states and to explore the generation, through processes like the ones considered here, of other forms of quantum fermonic correlations, such as the ones advanced in [35], based on a fermonic generalization of the measurement induced disturbances approach to quantum correlations proposed by Luo in [36]. Any further developments along these or related directions will be very welcome.

\section{Acknowledgments}

A.P.M, and P.A.B. acknowledge the Brazilian agencies MEC, MCTI, CAPES, CNPq, and FAPs for the financial support through the BJT Ciência sem Fronteiras Program. P.A.B. acknowledges support from the Spanish project grants FIS2014-59311-P (cofinanced by FEDER). A.V.H. gratefully acknowledges financial support from DGAPA, UNAM through project PAPIIT IA101816.

[1] G.C. Ghirardi, L. Marinatto, T. Weber, J. Stat. Phys. 108, 49 (2002).

[2] K. Eckert, J. Schliemann, D. Bruss, M. Lewenstein, Ann. Phys. (N.Y.) 299, 88 (2002).

[3] P. Zanardi, Phys. Rev. A 65, 042101 (2002).

[4] Y. Shi, Phys. Rev. A 67, 024301 (2003).

[5] G.C. Ghirardi, L. Marinatto, Phys. Rev. A 70, 012109 (2004).

[6] P. Levay, S. Nagy, and J. Pipek, Phys. Rev. A 72, 022302 (2005).

[7] G.C. Ghirardi, L. Marinatto, Optics and Spectroscopy 99, 386 (2005).

[8] J. Naudts, T. Verhulst, Phys. Rev. A 75, 062104 (2007).

[9] A. Plastino, D. Manzano, and J. Dehesa, Europhys. Lett. 86, 20005 (2009).

[10] A. Borras, A.R. Plastino, M. Casas, A. Plastino, Phys. Rev. A 78, 052104 (2008).

[11] C. Zander, A.R. Plastino, Phys. Rev. A 81, 062128 (2010).

[12] L. Amico, R. Fazio, A. Osterloh, V. Vedral, Rev. Mod. Phys. 80, 517 (2008).

[13] R. Paškauskas and L. You, Phys. Rev. A 64, 042310 (2001).

[14] A.D. Gottlieb and N.J. Mauser, Phys. Rev. Lett. 95, 123003 (2005).

[15] M.R. Dowling, A.C. Doherty, and H.M. Wiseman, Phys. Rev. A 73, 052323(2006).

[16] Y. S. Li, B. Zeng, X. S. Liu, and G. L. Long, Phys. Rev. A 64, 054302 (2001).

[17] M. C. Tichy, F. Mintert, A. Buchleitner, J. Phys. B: At. Mol. Opt. Phys. 44, 192001 (2011).

[18] P. Koscik, A. Okopinska, Few-Body Syst. 55, 1157 (2014).

[19] R.O. Esquivel, S. Lopez-Rosa, J.S. Dehesa, EPL 111 , 40009 (2015).

[20] M. Molina-Espiritu, R.O. Esquivel, S. Lopez-Rosa, J.S. Dehesa, Journ. Chem. Theo. Comp. 11, 5144 (2015).

[21] J.L. Birman, R.G. Nazmitdinov, and V.I. Yukalov, Phys. Rep. 526, 1 (2013).

[22] N.S. Simonovic, R.G. Nazmitdinov, Phys. Rev. A 92 , 052332 (2015).

[23] A.A. Melnikov and L.E. Fedichkin, Nature Sci. Rep. 6, 34226 (2016).

[24] P. Zanardi and X. Wang, J. Phys. A 35, 7947 (2002).

[25] J.R. Gittings and A.J. Fisher, Phys. Rev. A 66, 032305.

[26] H.M. Wiseman and J.A. Vaccaro, Phys. Rev. Lett. 91, 097902 (2003).

[27] N. Killoran, M. Cramer, M. B. Plenio, Phys. Rev. Lett 112, 150501 (2014). 
[28] D. Cavalcanti, L. M. Malard, F. M. Matinaga, M. O. Terra Cunha, M. F. Santos, Phys. Rev. B 76, 113304 (2007).

[29] J. Schliemann, D. Loss, and A. H. MacDonald, Phys. Rev. B 63, 085311 (2001).

[30] S.D. Barlett and H.M. Wiseman, Phys. Rev. Lett. 91, 097903 (2003).

[31] M. P. Strzys and J. R. Anglin, Phys. Rev. A 81, 043616 (2010).

[32] M. C. Tichy, J. Phys B: At. Mol. Opt. Phys. 47, 103001 (2014).

[33] A. Valdés-Hernández, A. P. Majtey, A. R. Plastino, Phys. Rev. A 91, 032313 (2015).

[34] A. P. Majtey, P. A. Bouvrie, A. Valdés-Hernández, A. R. Plastino, Phys. Rev. A 93, 032335 (2016).

[35] A.P. Majtey, C. Zander, and A.R. Plastino, Eur. Phys. Journ. D 67, 79 (2013).

[36] S. Luo, Phys. Rev. A 77, 022301 (2008). 